\title{
A Research Paper on Comparison between Energy Efficient Routing Protocol with Energy and Location in MANET
}

\author{
Ruchi Gupta ${ }^{1}$, Akhilesh A.Waoo ${ }^{2}$, Sanjay Sharma ${ }^{3}$ and P. S Patheja ${ }^{4}$ \\ ${ }^{1}$ (Computer Science Engineering, BIST/ RGPV, India) \\ ${ }^{2}$ (Computer Science Engineering, BIST/ RGPV, India) \\ ${ }_{3}^{3}$ (Computer Science Engineering, MANIT, India) \\ ${ }^{4}$ (Computer Science Engineering, BIST/ RGPV, India)
}

\begin{abstract}
Energy is an important issue in MANET (Mobile Ad hoc Network). Nodes in network are working in presence of limited energy. So, energy efficient routing is needed for reducing energy consumption. Energy efficient routing schemes can greatly reduce energy consumption and extends the lifetime of the networks. Multipath routing protocol (AOMDV) having more than one path, if the first one is break then second provides the proper packet delivery, this causes reduced node energy consumption and the topology of MANETs always changes, due to the high mobility of nodes. This makes it more difficult to find the routes that message packets use when they are routed. The location based routing provides the location information of the destination to route a packet toward. This paper provides comparison and study on reduced energy consumption by using multipath protocol and performance which is evaluated by performance metrics in case of AOMDV and energy based AOMDV. Now here DREAM protocol is used with AOMDV to finding location of mobile nodes but not with energy but here measures their performance with both multipath protocols.
\end{abstract}

Keywords - AOMDV, Dream, Energy, MANET, Multipath

\section{INTRODUCTION}

A mobile ad hoc network (MANET) is a dynamic distributed system of mobile formed by means of multi-hop wireless communication without the use of any existing network infrastructure. MANET routing protocols can be classified into two classes. Proactive protocols, they requires the nodes to periodically exchange the table information to update the pre-determine routes between any pair of source destination nodes. Reactive protocols can establish routes only when they require. MANET routing protocols can be classified on the bases of the methods of delivery of data packets from source to destination. Single Path routing protocols learn routes and select a single best route to each destination. These protocols are incapable of load balancing traffic. Multi-path routing protocols learn routes and can select more than one path to a destination. These protocols are better for performing load balancing. This paper provides the comparison between results about Multipath routing in AOMDV [13], AOMDV with location and Energy with AOMDV.

\section{OVERVIEW OF SELECTEd Routing Protocols \\ 2.1. AD-HOC ON-DEMAND MULTI PATH DISTANCE VECTOR ROUTING (AOMDV)}

One of the most commonly used AOMDV [1] is a multipath routing protocol provides loop-free extension to another multipath routing protocol AODV [12]. It ensures about disjoint alternate paths at every node, so that it can achieves path disjointness without using source routing. AOMDV with a route tables contain a list of paths for each destination, to support multipath routing. All the paths have the same destination sequence number to a destination. All the routes with the old sequence number are removed, once a rout advertisement with higher sequence number is received. Two additional fields, hop count and last hop, are stored in the route table entry to help address respectively the problems of loop freedom and path disjointness. The loop freedom guarantee from AODV is no longer required here, because the multipath routing protocol implement multipath discovery. AOMDV having two table fields hop count field and last hop field, in which hop count field initialized once at the time of the first advertisement for that sequence number and contains length of the longest path for a specific destination sequence number. That's why hop count field remain unchanged till a path for a higher destination sequence number is received. To ensure disjointness of that path in the route table, a node discards a path advertisement that has either a common last hop or a common next hop as already stored in the route table.

\subsection{ENERGY WITH AOMDV OR ENERGY EFFICIENT AOMDV (AOMDV WITH ENERGY)}

Reason behind the enhancement of AOMDV to AOMDV with energy [2], is to discover energy efficient paths between each node by calculating residual energy of each node by the use of GPS (Global 
positioning system) [14] [15] select the path consisting of minimum nodal residual energy and on the basis of descending order of nodal residual energy select all the routes. After this selection, a new route with maximum residual energy is selected to forward rest of the data packets. These results in the improvement of the individual node's battery power consumption and enhance the entire network lifetime. Following steps involves in it:

2.2.1. Calculation of residual energy of each node for the route discovery process.

2.2.2. Calculation of the path with minimum nodal residual energy.

2.2.3. Selection of all the routes on the basis of descending value of nodal residual energy

2.2.4. Finally select the path with maximal nodal residual energy to forward the data packets.

\subsection{LOCATION WITH AOMDV (DREAM)}

DREAM [3] is a location-based routing protocol work for Ad-hoc networks. It stands for Distance Routing Effect Algorithm for Mobility. Here in this comparison distance and mobility plays an important role, so in our named as Distance Routing Effect Algorithm for Mobility (DREAM) protocol for ad hoc networks. DREAM protocols have some desirable properties of providing bandwidth and energy efficiency. We can say that with respect to existing protocols, in DREAM more bandwidth and energy (required for transmission in each mobile node) can be used for the transmission of data messages. Most importantly:

2.3.1. The rate of control message generation is determined and optimized according to the mobility rate of each node individually.

2.3.2. Due to the "distance effect" the number of hops (radius from the moving node) it will be allowed to travel in the network before being discarded will only depend on the relative (geographic) distance between the moving node and the location tables being updated.

DREAM protocol provide loop-free path, since each data message propagates away from its source in a specific direction. DREAM protocol is also adaptive to mobility, since the frequency with which the location information is disseminated depends on the mobility rate.

\section{LITERATURE SURVEY}

There are various previous work have been done in the field of Efficient energy in AOMDV, for the improvement of Multipath routing with energy efficient techniques.

The researchers evaluates the performance of different AD-HOC routing protocols [2 ]such as DSDV, AODV, TORA, DSR and AOMDV in terms of energy efficiency and it also suggests a new routing algorithm that modifies AOMDV and also provides better performance compared to all the above protocols.

Pariza Kamboj and Ashok.K.Sharma,[4] research on the concept of local connectivity technique and preventive route reconfiguration on the basis of the current status of the nodes are being proposed that attempts to improve the performance and reliability in terms of reduced overhead, power and bandwidth requirement. These techniques also ensure good reduction in latency in case of link breakages and prevention of the network from splitting. The Energy Efficient Routing Multicast Protocol for MANET with Minimum Control Overhead is compared with other shared tree multicast protocol i.e. MAODV. Comparison was made on various parameters like Energy Consumption, Packet Delivery Ratio, Delay, and Throughput.

Azizol Abdullah et.al [6] produce the result of simulation done in identifying suitable ad hoc routing protocol which can be used for the target mobile grid application. Here is a simulation, showing comparison between three ad hoc routing protocols named as DSDV, DSR and AODV. This research mainly targets on the performance comparison based on packet delivery fraction and normalized routing load. According to researchers in future, extensive complex simulations could be carried out in gain a more in-depth performance analysis of the ad hoc routing protocols.

Shwaita Kodesia et.al, proposed a mobile ad hoc network (MANET) [7] consists of autonomous mobile nodes, each of the mobile nodes communicates directly with the nodes within its wireless range as well indirectly connected with other nodes in a network. An efficient routing protocol is required to discover routes between mobile nodes, in order to facilitate secure and reliable communication within a MANET. Due to the many advantages and different application areas of MANET, this field is rapidly growing. Security and energy efficiency are some challenges faced in MANETs, especially in designing a routing protocol. Here this is surveyed a number of energy efficient routing protocols and secure routing protocols. Here the study says, these protocols have different strengths and drawbacks.

Here the author Yun Ge et.al proposed a node-disjoint multipath routing protocol GMR [8] with the group mobility model. This protocol adopts two different approaches of routing intra-group routing and intergroup routing to adapt in two situations: within a group and among groups. Intra-group routing technique uses a proactive method. And Intergroup routing technique uses a reactive method with the zoning method, which uses 
dynamic topologies, and limits the region of broadcasting RREQ packets. The GMR protocol, provide group mobility model with good scalability in large and dense MANETs.

Farukh Mahmudur Rahman, Mark A Gregory, proposes a new MANET routing algorithm [9] showing quadrant based opportunistic routing, an intelligent energy matrix and energy status request messages with packet receipt acknowledgement notification. This algorithm uses an intelligent energy matrix to creates a look up table with the key characteristics: reputation value, residual battery level and energy consumption. This algorithm also balances the traffic uniformly across four intermediate nodes in any desired quadrant. The simulation shown here demonstrates the inclusion of the energy matrix and quadrant based routing, the number of broadcast messages decreases, reducing data flooding, providing improved channel efficiency and improves bandwidth utilization. To provide improved route stability, load balancing also increases the lifetime of intermediate nodes.

Here, Hua Chen, Hui Xu et.al compare the performance of various protocols for ad hoc networks [10]. Multipath routing is based on fresnel zone routing (FZR), and Energy aware Node Disjoint Multipath Routing (ENDMR) protocol. Simulations result show that, with the proposed network coding in ad hoc network multipath routing protocol (NC-MR), packet delivery ratio, network lifetime and packet loss could be improved in some of the cases.

Pariza Kamboj et.al constructs a shared bi-directional multicast tree [11] for its routing operations rather than a mesh, which helps in achieving more efficient multicast delivery. This shows the concept of small overlapped zones around each node for proactive topology maintenance within the zone. This algorithm implements a protocol which depends on the location information obtained using a distributed location service, which reduces shared multicast tree maintenance and overheads for route searching. This research provides a new technique of local connectivity management that attempts to improve the performance and reliability.

\section{SIMULATION ENVIRONMENT}

NS2 is an open-source event-driven simulator designed specifically for research in computer communication networks. The simulator we have used to simulate the ad-hoc routing protocols in is the Network Simulator 2 (ns) [16] from Berkeley. To simulate the mobile wireless radio environment we have used a mobility extension to ns that is developed by the CMU Monarch project at Carnegie Mellon University. Since its inception in 1989, NS2 has continuously gained tremendous interest from industry, academia, and government. On the basis of simulation parameters given in Table 2 simulation has been done in ns- 2 simulator.

\subsection{Simulation PARAMETERS}

Results are measured according to given simulation parameters shown in table 1.

TABLE I

Simulation Parameters for Case Study

\begin{tabular}{|l|l|}
\hline Simulator Used & NS- 2.31 \\
\hline Number of nodes & 25 \\
\hline Dimension of simulated area & $800 \mathrm{~m} \times 600 \mathrm{~m}$ \\
\hline Routing Protocol & AOMDV \\
\hline Other protocol & DREAM \\
\hline Simulation time & $100 \mathrm{sec}$. \\
\hline Traffic type (TCP \& UDP) & CBR $(3 \mathrm{pkts} / \mathrm{s})$ \\
\hline Packet size & 512 bytes \\
\hline Number of traffic connections & 6 \\
\hline Node movement at maximum Speed & random $(30 \mathrm{~m} / \mathrm{s})$ \\
\hline Transmission range & $250 \mathrm{~m}$ \\
\hline Transmission Energy Consumption & 1.5 joules \\
\hline Receiving Energy Consumption & 1 joules \\
\hline Idle Energy Consumption & 0.01 joules \\
\hline Sleep Energy Consumption & 007.0 joules \\
\hline
\end{tabular}

\subsection{Performance EVAluation}

4.2.1. PACKET DELIVERY RATIO: 
Ratio of the data packets received at the destination nodes to the packets that were sent by the sources.

\subsubsection{END TO END DELAY:}

Includes all the delays encountered by the packet at the different hops from the time it was sent by the source until the time it was received at the destination.

4.2.3. ROUTING LOAD: Number of routing packets (and supporting protocol control packets) transmitted per data packet delivered at the destination.

\subsubsection{THROUGHPUT:}

Number of data packets sends in per unit of time. Consider in per second.

\subsection{RESULTS}

In this section we present a set of simulation experiments to evaluate this protocol by comparing with the original energy based AOMDV routing protocol. Results are obtained after doing simulation of both previous and proposed scheme. Here we also include the performance of DREAM protocol.

\subsubsection{PACKeT LOSS ANALYSIS}

This graph represents the packet loss analysis clearly show in location based routing (packetLo_lost.tr) the packet loss is minimum as compare to normal AOMDV(packet_aomdv_lost.tr) and energy based AOMDV(Epacket_lost.tr). But here the main observation in between AOMDV, energy based AOMDV and location based AOMDV routing protocol. Here normal multipath routing has loss maximum number of packets. In the absence of energy factor the normal routing has been done as according to multipath routing protocol but after applying energy factor that always first calculate remaining energy of nodes then routing will done on the basis of shortest path and hop count then packet loss is minimum in energy based AOMDV.

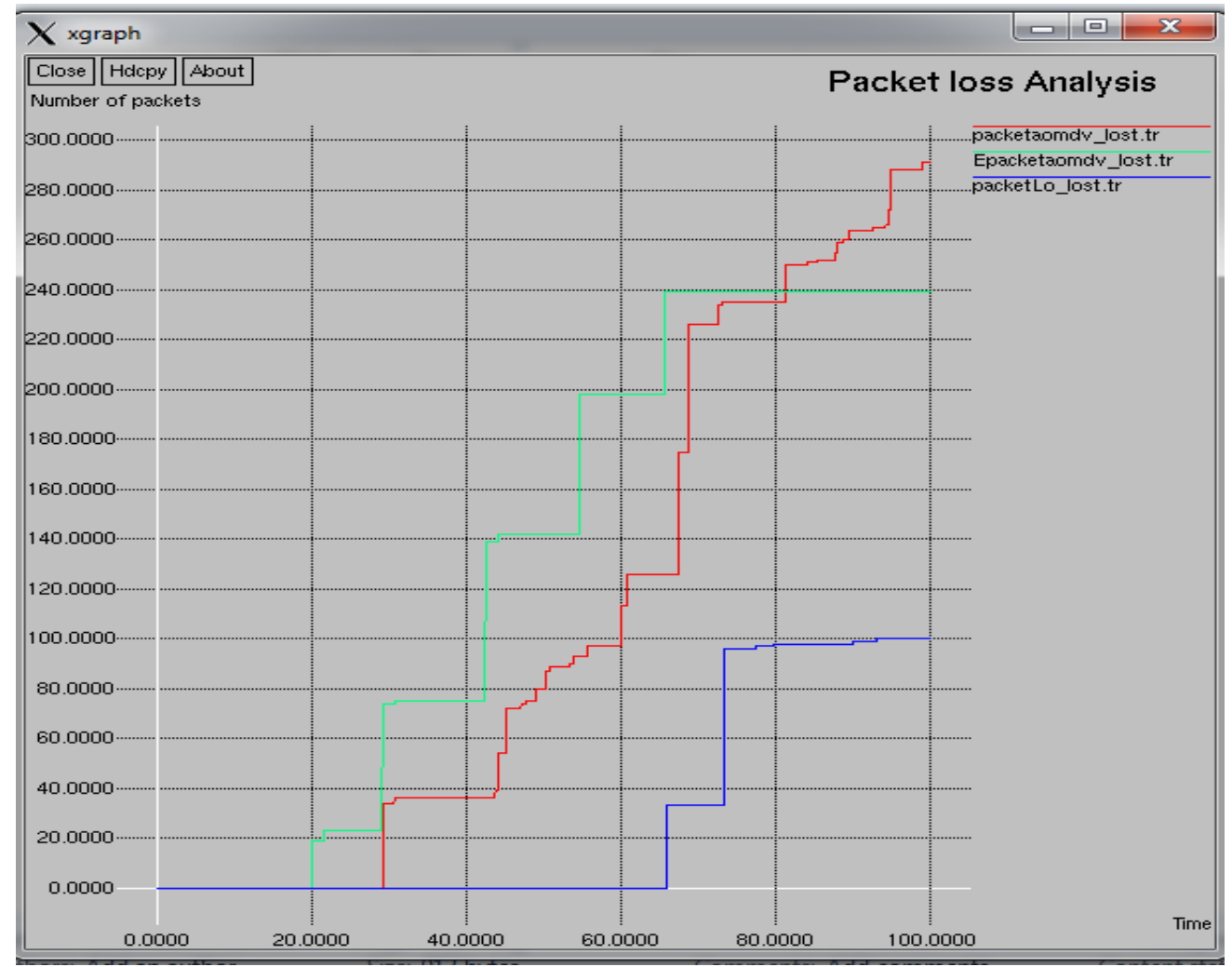

Fig.1 Packet loss analysis

\subsubsection{PACKet Delivery FraCtion ANALYSis(PDF)}

Packet delivery fraction is the one of the important performance parameter this represents the packets percentage successfully delivered to destination. Here the packet percentage in case of location based routing is maximum because nodes are aware about the location of destination and due to the presence of multipath routing protocol alternative route is always present then definitely PDF is more as compare to AOMDV and energy based AOMDV. 


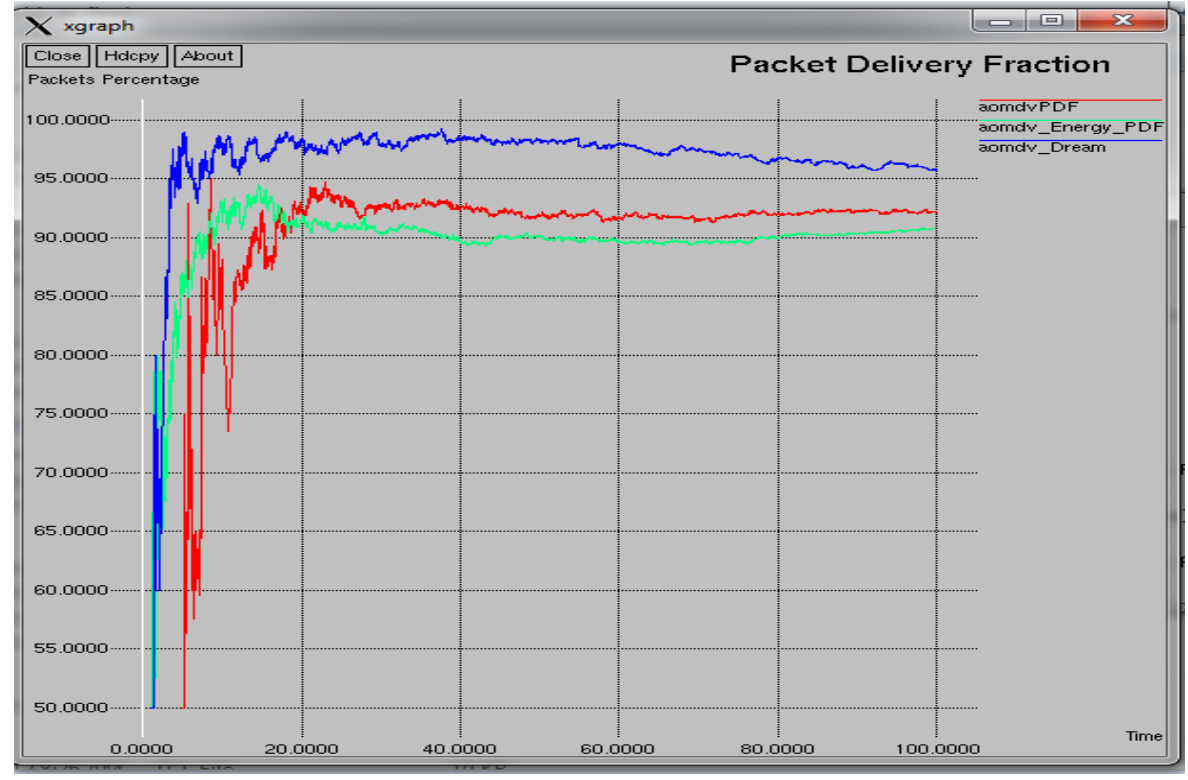

Fig .2 Packet Delivery Fraction analysis

\subsubsection{ROUTING LOAD ANALYSIS}

In routing load analysis we observe that in energy based AOMDV protocol the routing load is maximum it means that here links are break rapidly by that again connection request packets or control packets are generated by sender then routing load increases and route disjoint path are also not able to provide strong connection between sender and receiver but in normal multipath routing in the absence of energy factor routing load is minimum because one link is break then second one is present to providing proper data delivery. Now third case in DREAM protocol nodes are also forwarding data on the basis of location means senders are aware about the position of destination with multiple routes (because of AOMDV) then the routing load in location based routing is minimum as compare to all.

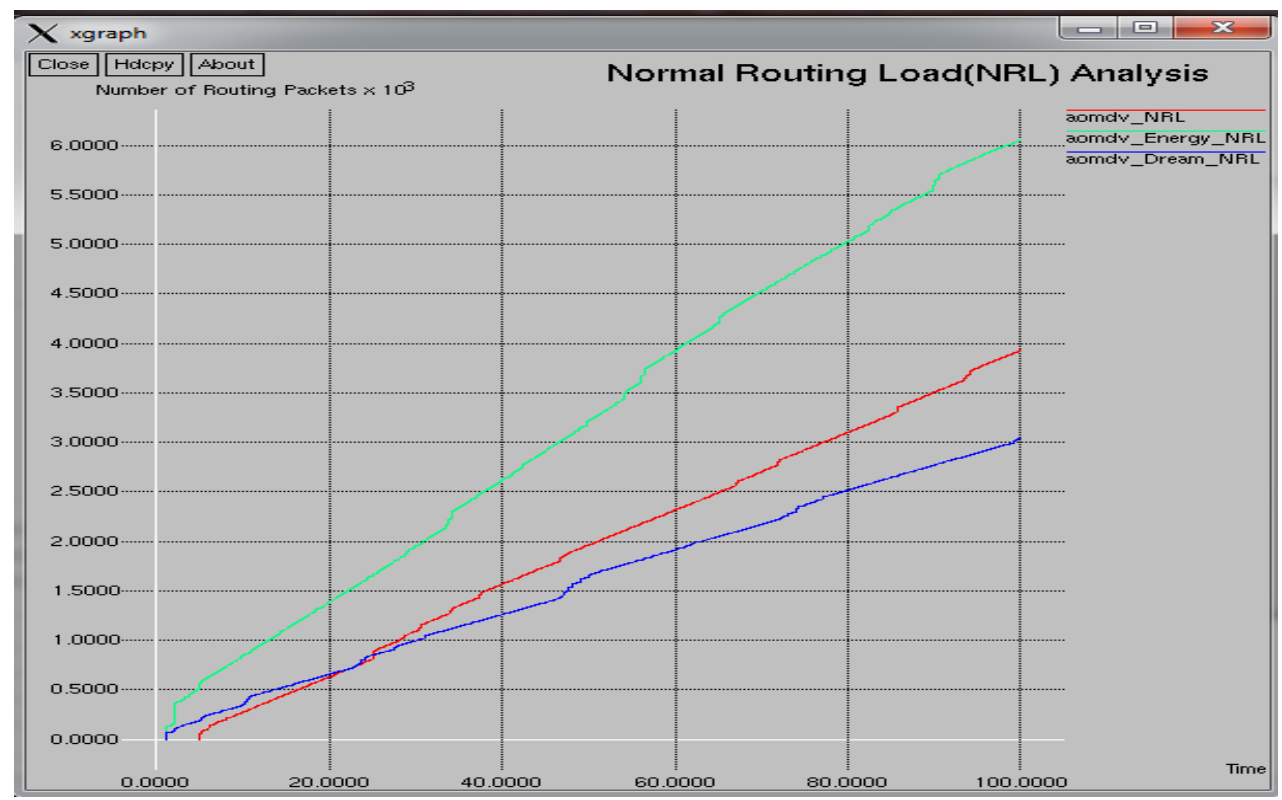

Fig. 3 Routing load analysis

\section{CONCLUSIONS}

In our simulations, we conduct the control overhead and the packet delivery rate with random mobility speeds. Simulation results show that the proposed location based multipath (AOMDV) can reduce the control overhead and increase the route lifetime than AOMDV. Only the forwarding neighboring nodes are involved in routing while the non-forwarding nodes are switched to idle state. This ensures reduction in energy consumption in the network. The results of DREAM location based protocol are very effective as compare to normal AOMDV routing and energy based AOMDV routing. Routing overhead and packet delivery fraction are shows 
excellent results with minimum packet loss. in location based multipath PDF are about $95 \%$ as compare AOMDV of about $92 \%$ and AOMDV with energy is about $90 \%$ with minimum routing overhead.

In future we also measure the performance of DREAM protocol with energy factor and compare the results with normal AOMDV location based routing. If the performance of energy based multipath routing protocol is better than existing three then definitely it reduces energy consumption and enhance network life time.

\section{Acknowledgements}

This research work could not have succeeded without the help and support of many people who truly care about nurturing the affective needs of students. First and foremost I would like to acknowledge the ongoing support of Dr. Sanjay Sharma and Prof. Akhilesh A. Waoo, Project Guide, BIST Bhopal in the design and implementation of the project. His suggestion about wireless networks in MANETs during the project was essential to the project's success.

\section{REFERENCES}

[1] Ruchi Gupta, Akhilesh A.Waoo and Dr. Sanjay Sharma, “A Survey of Energy Efficient Location Based Multipath Routing In MANET" in IJCA, Dec 2012.

[2] Jaya Jacob and V. Seethalakshmi, "EFFICIENCY ENHANCEMENT OF ROUTING PROTOCOL IN MANET" in IJAET, May 2012

[3] Stefano Basgani, Irnrich Chlamtac and Violet R. Syrotiuk, "A Distance Routing Effect Algorithm for Mobility (DREAM)".

[4] Pariza Kamboj, Ashok.K.Sharma, "Energy Efficient Multicast Routing Protocol for MANET with Minimum Control Overhead (EEMPMO)", in IJCT, Oct 2010.

[5] H.Vijayakumar, M.Ravichandran "Efficient Location Management of Mobile Node in Wireless Mobile Ad-hoc Network" in Proceedings of the National Conference on Innovations in Emerging Technology-2011.

[6] Azizol Abdullah, Norlida Ramly, Abdullah Muhammed, Mohd Noor Derahman, "Performance Comparison Study of Routing Protocols for Mobile Grid Environment", in IJCSNS, February 2008.

[7] Shwaita Kodesia and Asst. Prof. Prem Narayan Arya, "A REVIEW OF ENERGY EFFICIENT ROUTING PROTOCOLS FOR MOBILE AD-HOC NETWORK”, in JGRCS, May 2012.

[8] Yun Ge, Guojun Wang, and Jie Wu, "Node-Disjoint Multipath Routing with Group Mobility in MANETs", IEEE 2010.

[9] Farukh Mahmudur Rahman, Mark A Gregory, "Quadrant Based Intelligent Energy Controlled Multicast Algorithm for Mobile Ad Hoc Networks", ICACT2011.

[10] Hua Chen, Hui Xu, Ting Zhang, Baolin Sun, "Performance of Network Coding in Ad Hoc Network Multipath Routing Protocol", IEEE 2012.

[11] Pariza Kamboj, A.K.Sharma, "Scalable Energy Efficient Location Aware Multicast Protocol for MANET (SEELAMP)", JOURNAL OF COMPUTING, May 2010.

[12] C.E. Perkins, E.M. Royer and S.R. Das, “Ad hoc On Demand Distance Vector Routing,” Internet Draft: draftietf-manet-aodv-09.txt, November 2001.

[13] M. K. Marina and S. R. Das, "Ad hoc On-demand Multipath Distance Vector Routing," Computer Science Department, Stony Brook University, 2003.

[14] NAVSTAR GPS operations, available at URL: http://tycho.usno.navy.mil/gpsinfo.html.

[15] B.W. Parkinson and S.W. Gilbert, NAVSTAR: global positioning system - ten years later, Proceedings of the IEEE 71(10) (1983).

[16] Fast NS-2 simulator website http://lst.inf.ethz.ch/fast-ns2/. 